ARTICLE

https://doi.org/10.1038/s41467-019-09429-x

\title{
Excitation and coherent control of spin qudit modes in silicon carbide at room temperature
}

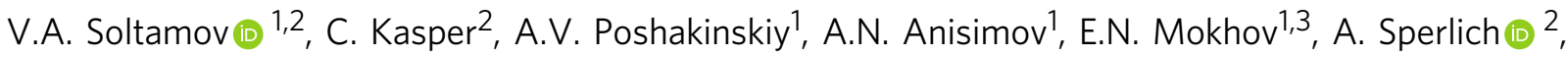 \\ S.A. Tarasenko1, P.G. Baranov ${ }^{1,4}$, G.V. Astakhov (10 ${ }^{1,2} \&$ V. Dyakonov (D) ${ }^{2}$
}

One of the challenges in the field of quantum sensing and information processing is to selectively address and coherently manipulate highly homogeneous qubits subject to external perturbations. Here, we present room-temperature coherent control of high-dimensional quantum bits, the so-called qudits, associated with vacancy-related spins in silicon carbide enriched with nuclear spin-free isotopes. In addition to the excitation of a spectrally narrow qudit mode at the pump frequency, several other modes are excited in the electron spin resonance spectra whose relative positions depend on the external magnetic field. We develop a theory of multipole spin dynamics and demonstrate selective quantum control of homogeneous spin packets with sub-MHz spectral resolution. Furthermore, we perform two-frequency Ramsey interferometry to demonstrate absolute dc magnetometry, which is immune to thermal noise and strain inhomogeneity.

\footnotetext{
${ }^{1}$ Ioffe Institute, St. Petersburg 194021, Russia. ${ }^{2}$ Experimental Physics VI, Julius-Maximilian University of Würzburg, Würzburg 97074, Germany. ${ }^{3}$ National Research University of Information Technologies, Mechanics and Optics, St. Petersburg 197101, Russia. ${ }^{4}$ Peter the Great St. Petersburg Polytechnic University, St. Petersburg 195251, Russia. Correspondence and requests for materials should be addressed to G.V.A. (email: astakhov@physik.uni-wuerzburg.de) or to V.D. (email: dyakonov@physik.uni-wuerzburg.de)
} 
Q uantum bit or qubit is a two-level system, which builds the foundation for quantum computation, simulation, communication, and sensing ${ }^{1}$. Quantum states of higher dimension, i.e., qutrits $(D=3)$ and especially qudits $(D=4$ or higher), offer significant advantages. Particularly, they can provide noise-resistant quantum cryptography ${ }^{2}$, simplify quantum $\operatorname{logic}^{3}$, and improve quantum metrology ${ }^{4}$. Flying and solid-state qudits have been implemented on the basis of photonic chips ${ }^{5}$ and superconducting circuits $^{6}$, respectively. The extension of the superconducting phase qubit to $D=5$ were used to emulate the dynamics of single spins $S=1 / 2,1$, and $3 / 2$, allowing a measurement of the Berry phase under $2 \pi$ rotation and paving a way to complex quantum computational protocols and architectures. However, superconducting phase qudits operate in the millikelvin temperature range. Therefore, there is still a lack of roomtemperature qudits with long coherence time.

To overcome this challenge, we have considered optical active centers in solids. Up to now, only two materials have been known to host the centers with spin and optical properties allowing coherent control and manipulation of their ground state spins by means of the optical and radiofrequency quanta at ambient condition. Namely, diamond containing negatively charged nitrogen-vacancy $\left(\mathrm{NV}^{-}\right)$centers and silicon carbide ( $\left.\mathrm{SiC}\right)$ containing silicon-carbon divacancies or negatively charged silicon vacancies $\left(\mathrm{V}_{\mathrm{Si}}\right)$ centers $^{7-9}$. Commercially produced wafer-scale $\mathrm{SiC}$ possesses ideal combination of hardness, radiation stability, and thermal conductivity of diamond, with the semiconducting properties and technological maturity of silicon ${ }^{10}$. In addition, the polymorphism of $\mathrm{SiC}$ enables the tuning of magnetic and optical characteristics of the centers ${ }^{11,12}$.

The divacancy centers posses the triplet ground state $(S=1)$, similarly to the $\mathrm{NV}^{-}$centers in diamond. They have been recently used to demonstrate a variety of prominent examples of the quantum phenomena in solids 8,13 , including the quantum entanglement in a macroscopic spin ensemble at ambient conditions ${ }^{14}$. Despite the unique properties, the triplet ground states of both the $\mathrm{NV}^{-}$centers in diamond and divacancies in $\mathrm{SiC}$ impose limitations on their use as qudits.

These limitations are lifted for the centers with the $S=3 / 2$ $(D=4)$ ground state, i.e., for the negatively charged silicon vacancy $\left(\mathrm{V}_{\mathrm{Si}}\right)$ centers in $\mathrm{SiC}$. Due to stable single-photon emission in NIR range, moderate zero-field splitting in the $\mathrm{MHz}$ region, zero-phonon lines with high Debay-Waller factor (up to $40 \%$ ), and long spin coherence times up to $20 \mathrm{~ms}$ at cryogenic temperatures, $\mathrm{V}_{\mathrm{Si}}$ centers were proposed for various quantum applications ${ }^{8,9,15-17}$. However, until now these centers have been treated as a canonical $S=1 / 2$ qubit system ${ }^{9,15-19}$.

Here, we develop a concept of operating $\mathrm{V}_{\mathrm{Si}}$ centers as qudits, which can reside in multiple superpositions of four basis states in the Hilbert space with the spin projections $m_{\mathrm{S}}= \pm 3 / 2, \pm 1 / 2$. Similar to atomic orbitals, an ensemble of such spin qudits is described by 15 linearly independent spherical multipoles, with three components being the spin dipole $\mathcal{P}$, five components being the spin quadrupole $\mathcal{D}$, and seven components being the spin octupole $\mathcal{F}^{20}$. Examples of such multipoles and their description in terms of the spin-density matrix $\rho$ are sketched in Table 1. When qudit multipoles are excited, they decay with three relaxation times: that of the spin dipole $\left(T_{p}\right)$, quadrupole $\left(T_{d}\right)$, and octupole $\left(T_{f}\right)$.

We use this concept to experimentally excite the qudit modes at ambient conditions by means of two-frequency optically detected magnetic resonance (ODMR). Strikingly, we found their spectral width to be about an order of magnitude narrower than the inhomogeneous broadening of the corresponding spin resonance. By applying the Ramsey interferometry to these spin qudits, we achieve a spectral selectivity of $600 \mathrm{kHz}$ and a spectral resolution of $30 \mathrm{kHz}$. As a practical consequence, we demonstrate absolute DC magnetometry insensitive to thermal noise and strain fluctuations.

We focus on the $\mathrm{V}_{\mathrm{Si}}$ centers in two main hexagonal polytypes of $\mathrm{SiC}$, namely $6 \mathrm{H}$ and $4 \mathrm{H}$, with natural $\left({ }^{29} \mathrm{Si} 4.7 \%,{ }^{13} \mathrm{C} 1.1 \%\right)$ and modified ${ }^{29} \mathrm{Si} 1 \%,{ }^{13} \mathrm{C} 1.1 \%$ ) isotope abundance (see the Methods section). Exemplary electron paramagnetic resonance (EPR) spectrum of the investigated $6 \mathrm{H}^{2}{ }^{28} \mathrm{SiC}$ sample is shown in Supplementary Fig. 1a.

\section{Results}

Visualization of the spin qudit modes. To experimentally visualize the $\mathrm{V}_{\mathrm{Si}}$ spin qudit modes, we use optically detected magnetic resonance (ODMR) as described elsewhere 11,15,19,21,22 $^{1}$ (see also Methods and Supplementary Fig. 2). Optical pumping results in a preferential population of either the $m_{S}= \pm 3 / 2$ or $m_{S}= \pm 1 / 2$ states (depending on $\mathrm{SiC}$ polytype and the $\mathrm{V}_{\mathrm{Si}}$ crystallographic site, as was shown in refs. ${ }^{9,12,20,23}$ ). Such a spin alignment is theoretically described by the contributions to the diagonal components of the spin-density matrix $\delta \rho_{+3 / 2,+3 / 2}=$ $\delta \rho_{-3 / 2,-3 / 2}=-\delta \rho_{1 / 2,1 / 2}=-\delta \rho_{-1 / 2,-1 / 2}$. In the multipole decomposition of the spin density matrix, this corresponds to the appearance of the spin quadrupole $\mathcal{D}_{0}$ (see Table 1). Application of a strong microwave (MW) field at a fixed frequency $v_{\text {pump }}$ mixes the $m_{S}= \pm 3 / 2$ and $m_{S}= \pm 1 / 2$ states, resulting in the excitation of other qudit modes as well. They are probed as relative difference of the spin-dependent photoluminescence $\triangle \mathrm{PL} / \mathrm{PL}$, while sweeping the frequency of a second (weak) MW field $v_{\text {probe }}$.

Figure 1a shows an ODMR resonance associated with the V3 $\left(\mathrm{V}_{\mathrm{Si}}\right)$ center in $6 \mathrm{H}^{-28} \mathrm{SiC}^{23-25}$. The ODMR resonance without the pump MW field (black curve) reveals the zero-field spin splitting $2 D=26.8 \mathrm{MHz}$ between the Kramers doublets $\pm 1 / 2$ and $\pm 3 / 2$ states, whereas the sign of $D$ determining the ordering of the Kramers doublets, is negative ${ }^{12}$. Application of a strong, pump MW field at $v_{\text {pump }}=26.8 \mathrm{MHz}$ saturates the spin transitions, that is seen as a spectral hole burning ${ }^{26,27}$. To model it, we assume that the ODMR resonance is inhomogeneously broadened and agregates many homogeneous spin packets with different resonance frequencies, as schematically shown by thin lines in Fig. 1b. The pump MW field excites qudit modes in particular spin packets, which are detected as a reduction of the ODMR signal at certain frequencies. In zero magnetic field, all the excited modes are degenerated and manifest themselves as a single spectral hole. Remarkably, the spectral width of such hole can be much narrower than the inhomogeneous linewidth, and in certain cases we observe $250 \mathrm{kHz}$ (Supplementary Figs. 3, 4).

The two-frequency technique enables potentially the access to the properties of spin centers with the resolution determined by the natural linewidth of individual centers. At the same time, the detected signal is much stronger and less noisy than that from a single center, which is crucial for applications.

To increase the sensitivity, we modify the detection scheme. The MW pump is now modulated on/off, and the ODMR spectrum is detected using a lock-in amplifier. The pump-induced changes of the ODMR spectrum are presented in Fig. 1c for several magnetic fields $B_{z}$ applied along the $c$-axis of SiC. Several qudit modes $v_{\text {mod }}$ are now clearly detected and their spectral positions relative to $v_{\text {pump }}$ depend on $B_{z}$.

To understand the spectrum of spin qudit modes, we analyze successively the most probable sources of inhomogeneous broadening: (i) magnetic fluctuations (for instance, due to nuclear fields) and (ii) variations of the zero-field splitting (for instance, 


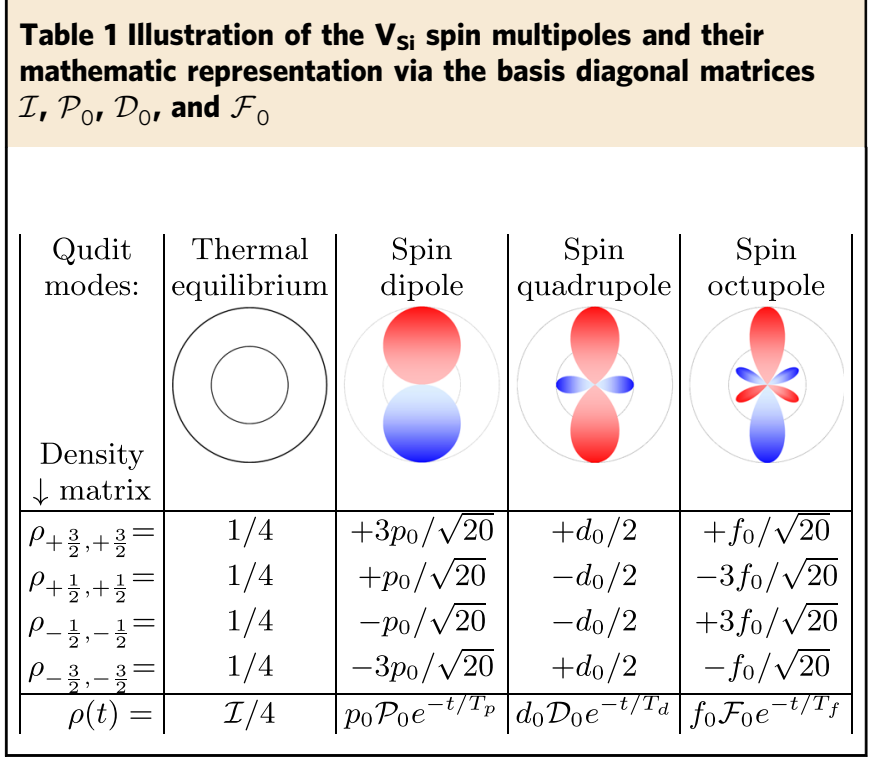

due to local strain). The first mechanism sufficiently influences the inhomogeneous broadening of the $\mathrm{NV}^{-}$center ODMR transitions in diamond, as shown in ref. ${ }^{26}$. Upon application of the magnetic field along the $c$-axis, a single spin packet is characterized by four resonances shifting linearly with the magnetic filed as $2 D \pm \gamma B_{z}$ and $2 D \pm 2 \gamma B_{z}{ }^{28}$. Here, $\gamma=28$ $\mathrm{MHz} / \mathrm{mT}$ is the gyromagnetic ratio. The corresponding fan chart is shown by red lines in Fig. 1d. The line thickness represents the coupling strength to the MW field. Another spin packet feels another local magnetic field $\delta B$ and the fan chart is shifted along the vertical axis in Fig. 1d. The mean value $\delta \bar{B}$ defines the inhomogeneous ODMR linewidth.

Assume that the pump MW field is in resonance with one of the spin transitions of a particular spin packet (the solid circle in Fig. 1d), then the other three spin resonances of the same spin packet will be effected due to the excitation and relaxation of spin multipoles, leading to the appearance of qudit modes (the open circles in Fig. 1d). Their spectral position relative to the pump frequency is symmetric in respect to the zero-field splitting $2 D$. In case when $v_{\text {pump }}=2 D$, the excited qudit modes remain degenerate even when $B_{z} \neq 0$ (the square in Fig. 1d), which contradicts to the experimental observation of Fig. 1c. Thus, we conclude that magnetic fluctuations are not the main source of inhomogeneous broadening.

We now consider the other mechanism of broadening, caused by the variation of the zero-field splitting around the mean value $2 \bar{D}=26.8 \mathrm{MHz}$. This mechanism is natural to expect, given that we investigate isotopically purified $6 \mathrm{H}_{-}{ }^{28} \mathrm{SiC}$ with low content of the spin-carrying ${ }^{29} \mathrm{Si}$ isotopes. In this case, the fan chart of the field-dependent ODMR lines associated with different spin packets are shifted along the horizontal axis in Fig. 1e. Positions of the excited qudit modes are given by $v_{\text {mod }}=v_{\text {pump }} \pm s \gamma B_{z}$ with $s=1,2,3$, and 4 . They depend on the magnetic field strength, which is in qualitative agreement with the experiment of Fig. 1c.

Figure $2 \mathrm{a}$ visualizes the magnetic field evolution of the $\mathrm{V} 3\left(\mathrm{~V}_{\mathrm{Si}}\right)$ spin qudit modes in $6 \mathrm{H}_{-}{ }^{28} \mathrm{SiC}$. The observed behavior is more complex compared with the qualitative consideration above. Not all qudit modes shift linearly with $B_{z}$, they have different strength and some of them are not even detected. To understand this behavior, we have developed a theory of qudit mode excitation in inhomogeneously broadened spin ensembles (see the Methods section).

We start from the effective spin Hamiltonian of the $\mathrm{V}_{\mathrm{Si}}$ centers in a simple axial model $H=D\left(S_{z}^{2}-5 / 4\right)+\gamma \mathbf{S} \cdot \mathbf{B}$. In small magnetic fields that we consider, $\gamma B \ll D$, the eigenstates are given by

$$
\begin{aligned}
& E_{ \pm 3 / 2}=+D \pm \frac{3}{2} \gamma B_{z}, \\
& E_{ \pm 1 / 2}=-D \pm \frac{1}{2} \gamma \sqrt{B_{z}^{2}+4 B_{\perp}^{2}},
\end{aligned}
$$

where $B_{z}$ and $B_{\perp}$ are the magnetic field components parallel and perpendicular to the $c$-axis, respectively. Due to the mixing of the spin states by $B_{\perp}$ and a low trigonal pyramidal local symmetry of the $\mathrm{V}_{\mathrm{Si}}$ centers in $\mathrm{SiC}$, all four transitions between the $\pm 1 / 2$ and $\pm 3 / 2$ spin states are allowed ${ }^{28}$. Their spectral positions read

$$
\nu_{\bmod }^{\left(s, s^{\prime}\right)}=\nu_{\text {pump }}+3 s \gamma B_{z}+s^{\prime} \gamma \sqrt{B_{z}^{2}+4 B_{\perp}^{2}},
$$

where $s, s^{\prime}=0, \pm 1$. Equation (2) describes the spectral hole burning at $v_{\text {pump }}\left(s, s^{\prime}=0\right)$ and 8 qudit modes.

We now analyze the strength of the qudit modes coupling to the MW field and how excitations between these modes are transfered. At room temperature, when the energy of thermally excited phonons is much higher than the spin splitting, one can neglect the anisotropy of spin relaxation and consider that all spin multipoles relax independently. If spin relaxation occurs due to fluctuating magnetic fields, only the transitions with $\Delta m_{S}= \pm 1$ are allowed, as had also been assumed in the earlier works ${ }^{15}$. Application of this constraint to the matrix of spin relaxation, see Eq. (15) in the Methods section, yields the ratio between the multipole relaxation times $T_{P}=3 T_{d}=6 T_{f}$. Remarkably, the relaxation rates $+3 / 2 \leftrightarrow+1 / 2$ and $-3 / 2 \leftrightarrow-1 / 2$ are not equal to that of $+1 / 2 \leftrightarrow-1 / 2$, and we obtain for them $(1 / 2) T_{d}^{-1}$ and $(2 / 3) T_{d}^{-1}$, respectively.

The analytical solution of the rate equations, Eq. (12) in Methods, shows that the strength of the modes $\left(s, s^{\prime}\right)=(+1,+1)$, $(+1,-1),(-1,+1)$, and $(-1,-1)$ are proportional to $5 T_{d}-T_{p}-$ $4 T_{f}$. Therefore, they vanish if the aforementioned ratio $T_{p}=$ $3 T_{d}=6 T_{f}$ holds. As a consequence, only four out of eight possible qudit modes are observed in Fig. 2 a. The evolution of the qudit modes with $B_{z}$, calculated as described in the Methods section, is shown in Fig. 2b. Here, we assume the Gaussian distribution of the zero-field splitting, $f(D) \propto \exp \left[(D-\bar{D})^{2} /\left(\delta D^{2}\right)\right]$, with the mean value $2 \bar{D}=26.8 \mathrm{MHz}$ corresponding to the $\mathrm{V} 3\left(\mathrm{~V}_{\mathrm{Si}}\right)$ center in $6 \mathrm{H}-\mathrm{SiC}$. A perfect agreement with the experimental data is achieved for $B_{\perp}=60 \mu \mathrm{T}$, accounting for a uncompensated perpendicular component of the external magnetic field.

Some discrepancy between the measured and calculated images may stem from more general form of spin relaxation, not described by a single parameter, or more general form of inhomogeneous broadening.

Coherent control of spin qudit modes. To further confirm the conclusions of our model, we investigate the qudit modes in a stronger magnetic field $B_{z}=211 \mu \mathrm{T}$ when the Zeeman splitting is larger than inhomogeneous broadening. A precise analysis of the ODMR spectra ${ }^{29,30}$ shows that $B_{\perp}=73 \mu \mathrm{T}$ remains almost the same. Figure 3 a shows relative positions of the $\mathrm{V}_{\mathrm{Si}}$ spin sublevels calculated for this field configuration. The ODMR spectrum consists of four lines [the lower curve in Fig. 3b] and the corresponding spin transitions enumerated from $v_{1}$ to $v_{4}$ are shown by arrows in Fig. 3a. The inner transitions $v_{1,2}$ are stronger than the outer transitions $v_{3,4}$, in accordance with earlier studies ${ }^{28}$.

Now, we burn a spectrally narrow hole at $v_{1}\left(v_{\text {pump }}=21.8\right.$ $\mathrm{MHz}$ ), as shown in Fig. 3b. As expected from our model, excitation of qudit modes leads to the emergence of spectral holes at the $v_{2}, v_{3}$, and $v_{4}$ transitions [see the upper curve in Fig. 3b]. Their spectral positions shift linearly with $v_{\text {pump}}$, keeping the frequency difference the same. This is particularly pronounced in another sample with much larger inhomogeneous broadening, 

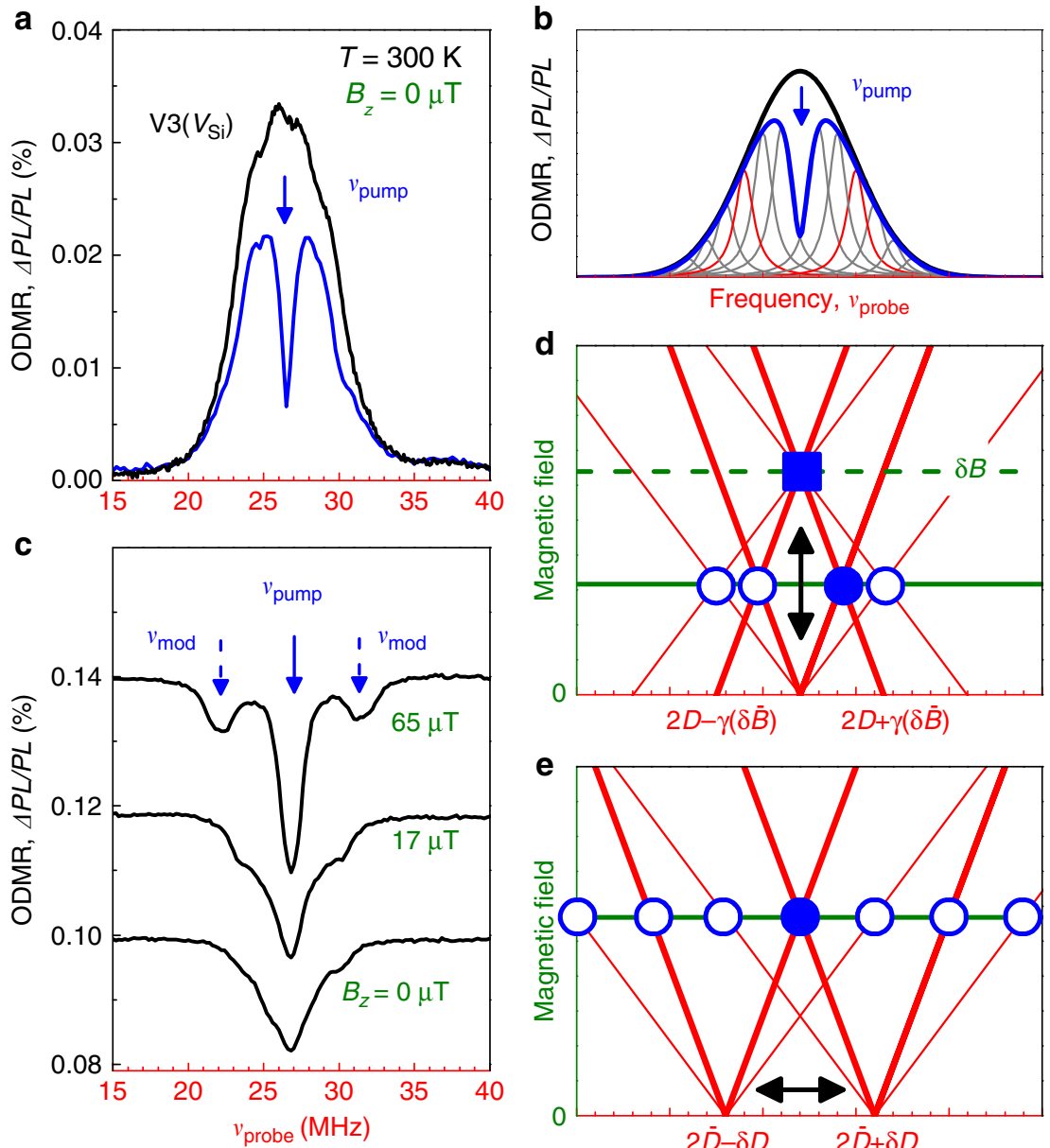

Frequency, $v_{\text {probe }}$
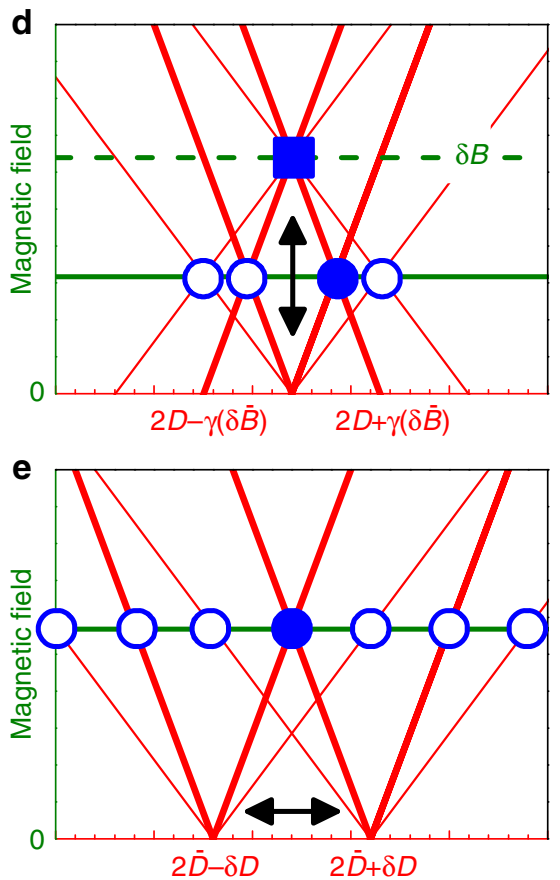

Fig. 1 Two-frequency ODMR spectroscopy. a ODMR spectrum of isotopically purified $6 \mathrm{H}-\mathrm{SiC}$ without and with the pump $\mathrm{MW}$ field at $v_{\text {pump }}=26.8 \mathrm{MHz}$. The pump and probe powers are $W_{\text {probe }}=7 \mathrm{dBm}$ and $W_{\text {pump }}=14 \mathrm{dBm}$, respectively. $\mathbf{b}$ A schematic modeling of (a) by subtraction the contribution of a homogeneously broadened spin packet from the Gauss-shaped ODMR line. c Pump-induced changes in the ODMR spectra in different magnetic fields. The dashed arrows indicate the qudit mode frequencies. $\mathbf{d}$, e The fan charts of the qudit modes as a function of the magnetic field in case when the inhomogeneous broadening is caused by magnetic fluctuations and variation for the zero-field splitting, respectively. The solid symbols correspond to the pump frequencies and the open circles correspond to the excited qudit mode frequencies. The red lines of the fan charts indicate the spectrally selected spin packets

isotopically purified $4 \mathrm{H}^{2}{ }^{28} \mathrm{SiC}$ (Supplementary Fig. 5). We hence conclude that by varying $v_{\text {pump }}$ within the $v_{1}$ ODMR line, different spin packets are selected. Remarkably, there is another signal at $v_{5}$ in Fig. $3 \mathrm{~b}$, which has the opposite sign and appears only if the $v_{1}$ qudit mode is excited. The same behavior is also observed in $4 \mathrm{H}-\mathrm{SiC}^{30}$ (see also Supplementary Fig. 6). These general properties are used to implement spectrally selective coherent control of qudit modes.

We start with driving the $v_{1}$ transition. Figure $3 \mathrm{c}$ shows Rabi oscillations when the driving power is relatively low $W_{1}=11$ $\mathrm{dBm}$. The corresponding $\pi$-pulse duration of $1.2 \mu$ s provides sub$\mathrm{MHz}$ spectral selectivity. We then spectrally select a spin packet using a long $\pi$ pulse at $v_{1}$ and coherently drive it at the $v_{5}$ resonance followed by a second long $\pi$ pulse at $v_{1}$ for the readout [inset of Fig. 3d]. A high driving power $W_{5}=33 \mathrm{dBm}$ yields fast Rabi oscillations, as presented in Fig. 3d. For such a power, the $\pi /$ 2 pulse is $80 \mathrm{~ns}$, corresponding to a bandwidth of $\sim 10 \mathrm{MHz}$. This bandwidth is wide enough to encompass the $v_{5}$ linewidth.

Two-frequency Ramsey interferometry. To highlight the advantages of qudit modes for quantum information processing and sensing, we demonstrate Ramsey-based absolute magnetometry performing two-frequency experiments with the protocol shown in Fig. 4a (and Supplementary Fig. 2). When the probe frequency $v_{\text {probe }}=6.7 \mathrm{MHz}$ is equal to the $v_{5}$ qudit mode frequency, the signal represents free induction decay (Fig. 4b). We note that though the other, $v_{3}$ resonance lies within the bandwidth, the population of the $m_{S}=+3 / 2$ and $m_{S}=-1 / 2$ is equal after the $v_{1} \pi$-pump pulse (Fig. $3 \mathrm{a}$ ), and this resonance is not driven. This is also confirmed by the absence of pronounced fringes in the free induction decay of Fig. $4 \mathrm{~b}$.

When $v_{\text {probe }}=11.7 \mathrm{MHz}$ is detuned from the $v_{5}$ mode, Ramsey fringes are clearly detected (Fig. 4c). A fit of these dynamics to $\cos \left(2 \pi f_{R} \tau\right) \exp \left(-\tau / T_{2}^{*}\right)$ gives the coherence time of spin packets $T_{2}^{*}=357 \pm 24 \mathrm{~ns}$. For comparison, we also perform standard Ramsey measurements using single MW frequency, but do not observed fringes because of inhomogeneous broadening and non-selective excitation (Supplementary Fig. 7).

Solid circles in Fig. 4d represent the fast Fourier transform (FFT) of the experimental data from Fig. 4c. The fitting of these data to a Lorentz function yields the frequency of the Ramsey fringes $f R=4.51 \pm 0.03 \mathrm{MHz}$, with the corresponding spectral resolution of $30 \mathrm{KHz}$. Following Eq. (1), the effective magnetic 


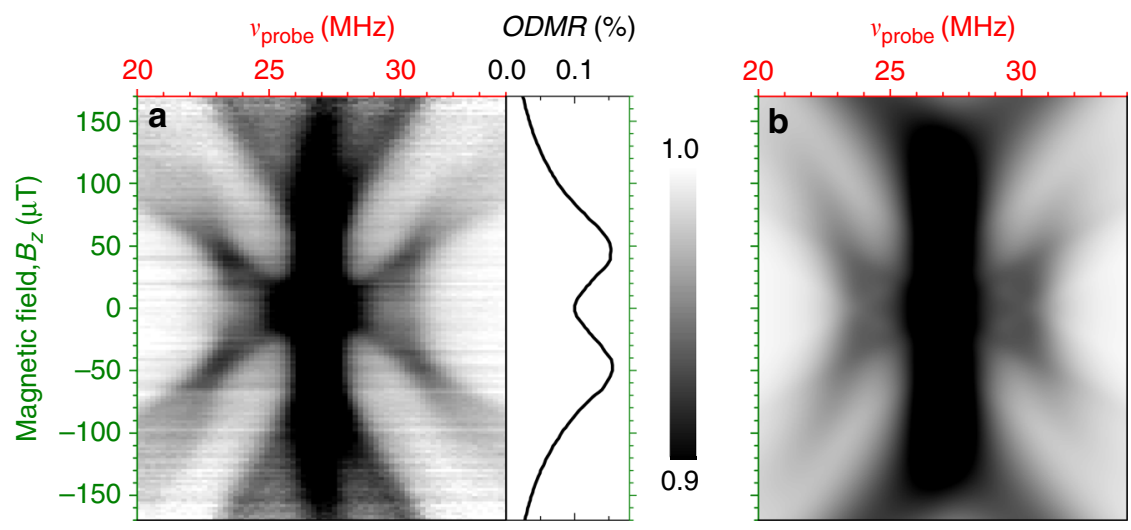

Fig. 2 Visualization of the excited qudit modes. a Magnetic field evolution of the qudit modes in an inhomogeneously broadened spin ensemble. The ODMR signal is normalized for each magnetic field. The base ODMR signal is presented on the right side. $\mathbf{b}$ Calculated evolution of the spin qudit modes assuming $T_{p}=3 T_{d}=6 T_{f}$ (see text for details)
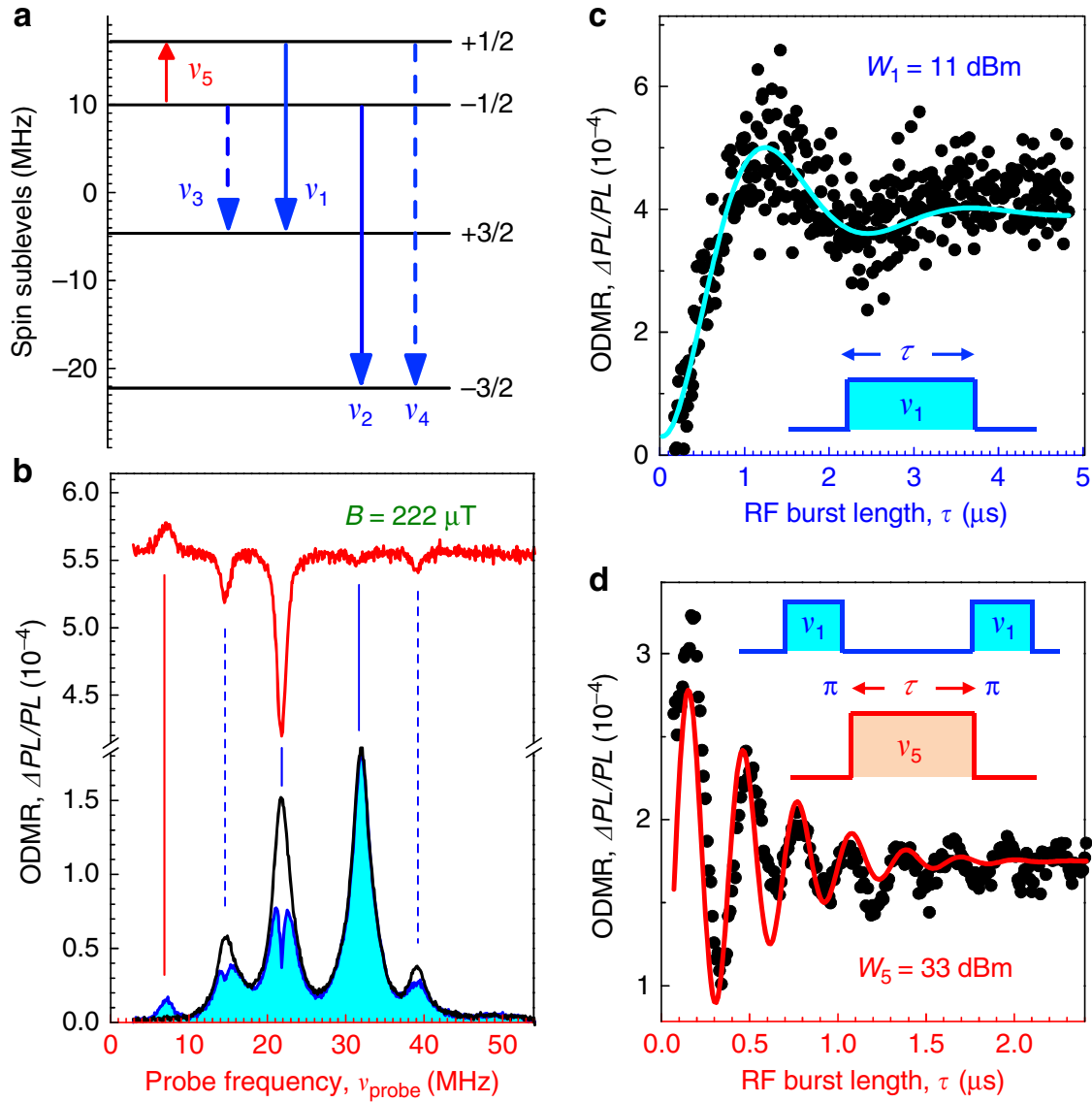

Fig. 3 Coherent manipulation of spin qudit modes. a MW-induced transitions between different spin sublevels after optical pumping into the $m_{S}= \pm 1 /$ 2 states. The solid and dashed arrows correspond to the transitions with large and small matrix elements, respectively. $\mathbf{b}$ ODMR spectra in a magnetic field of $222 \mu \mathrm{T}$ with and without MW pump at $v_{\text {pump }}=21.8 \mathrm{MHz}$. The upper curve shows the spectrum of the excited qudit modes. $\mathbf{c}$ Rabi oscillations at the $v_{1}$ resonance driven by the $\mathrm{MW}$ power $W_{1}=11 \mathrm{dBm}$ with the corresponding $\pi$-pulse duration of $1.2 \mu$ s. $\mathbf{d}$ Rabi oscillations of a spin packet at the $v_{5}$ resonance driven by the MW power $W_{5}=33 \mathrm{dBm}$

field can be measured with high accuracy as

$$
B_{\text {eff }}=\frac{\nu_{\text {probe }}-f_{\mathrm{R}}}{\gamma \sqrt{1+3 \sin ^{2}(\theta)}}
$$

Here, $\theta$ is the angle between the magnetic field direction and the $c$-axis of SiC. It is calculated from the ratio $\left(v_{2}-v_{1}\right) /\left(v_{4}-v_{3}\right)$ with resolution better than $1^{\circ}$ following the previously described procedure ${ }^{29}$, which yields $\theta=19^{\circ} \pm 0.5^{\circ}$. The gyromagnetic ratio $\gamma$ is the constant value. We then obtain from Eq. (3) $B_{\text {eff }}=223 \pm$ $2 \mu \mathrm{T}$. To analyze the spectral selectivity of different spin packets, we marginally vary the external magnetitic field strength and the pump/probe frequencies. The results are summarized in Table 2, 


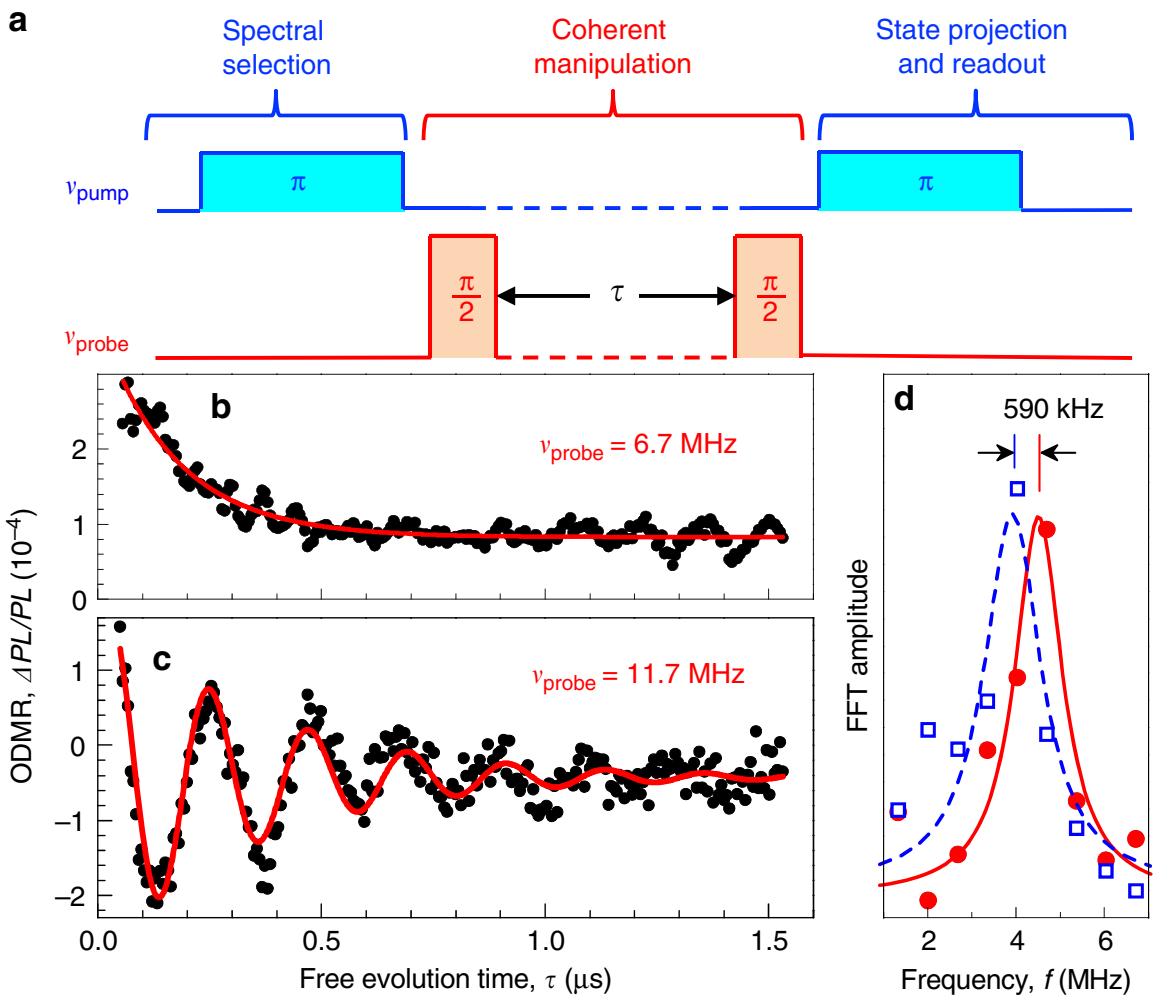

Fig. 4 Ramsey interferometry of spin qudit modes. a Pulse pattern for the spectral selection, coherent manipulation, and state projection followed by the readout of spin packets. $\mathbf{b}$ Ramsey measurement of the spin packet selected by $v_{\text {pump }}=21.8 \mathrm{MHz}$ in a magnetic field of $223 \pm 2 \mu \mathrm{T}$. The probe frequency $v_{\text {probe }}=6.7 \mathrm{MHz}$ is set to the $v_{5}$ resonance. The solid line represents the fit to an exponential decay with $T_{2}^{*}=168 \pm 7 \mathrm{~ns}$. c The same as (b) but the probe frequency $v_{\text {probe }}=11.7 \mathrm{MHz}$ is detuned from the $v_{5}$ resonance. The solid line represents the fit to an exponentially decaying sinusoid with $T_{2}^{*}=357 \pm 24 \mathrm{~ns}$. d FFT of the Ramsey fringes fitted to a Lorentz function. The solid line corresponds to the data from (c). The dashed line is for the same spin packet as in c but probed in a magnetic field of $242 \pm 3 \mu \top$

where measurement No. 1 corresponds to the case discussed above. We then increase $B$ and change $v_{\text {pump }}$ to select the same spin packet (measurement No. 2 in Table 2). The corresponding FFT of the Ramsey fringes is represented by open squares in Fig. $4 \mathrm{~d}$ and a lorentzian fit (the dashed line) yields $f_{R}=3.92 \pm$ $0.05 \mathrm{MHz}$ and $B_{\text {eff }}=242 \pm 3 \mu \mathrm{T}$. A comparison of measurements No. 1 and No. 2 demonstrates the spectral selectivity of spin packets to be $\sim 600 \mathrm{kHz}$. We note that an unusually narrow ODMR linewidth of $500 \mathrm{kHz}$ in $\mathrm{SiC}$ was observed earlier in twofrequency experiments, but its origin had not been discussed ${ }^{30}$.

\section{Discussion}

Measurements Nos. 13 in Table 2 demonstrate that the effective magnetic field $B_{\text {eff }}$ seen by different spin packets, which are selected by different $v_{\text {pump }}$, is the same within the error bars. This is a manifestation that the inhomogeneous broadening is caused mostly by local variations of the zero-field splitting $2 D$ rather than magnetic fluctuations. Remarkably, another pair of measurements (No. 2 and No. 3) in Table 2 show that the magnetic field strength can be measured without calibration of the zerofield splitting, which can be used to implement absolute (i.e., immune to thermal noise and strain inhomogeneity) DC magnetometry ${ }^{31}$.

To summarize, we demonstrated coherent manipulation of spin qudit modes in isotopically purified $\mathrm{SiC}$ at room temperature. We also developed a theory describing the excitation and detection of these modes in inhomgeneously broadened systems and showed that qudits are characterized by multiple relaxation times. These findings can lead to dipole-coupled networks ${ }^{11}$,

\begin{tabular}{|c|c|c|c|c|c|}
\hline $\begin{array}{l}\text { No. } \\
f_{R}\end{array}$ & $\begin{array}{l}I \\
(m A) \\
(M H z)\end{array}$ & $\begin{array}{l}B_{\text {eff }} \\
(\mu T)\end{array}$ & $\begin{array}{l}V_{\text {pump }} \\
(\mathbf{M H z})\end{array}$ & & $\begin{array}{l}V_{\text {probe }} \\
(M H z)\end{array}$ \\
\hline 1 & 100 & 21.80 & 11.70 & $\begin{array}{l}4.51 \pm \\
0.03\end{array}$ & $223 \pm 2$ \\
\hline 2 & 105 & 21.20 & 11.70 & $\begin{array}{l}3.92 \pm \\
0.05\end{array}$ & $242 \pm 3$ \\
\hline 3 & 100 & 21.20 & 11.70 & $\begin{array}{l}4.51 \pm \\
0.04\end{array}$ & $223 \pm 2$ \\
\hline
\end{tabular}

Experimental data are presented in Fig. 4 and Supplementary Fig. 8

unconditional electron-nuclear spin registers ${ }^{14}$, and spectral selection of highly coherent individual spins ${ }^{15,22,32}$, particularly in nanocrystals ${ }^{33}$. Our results hence open new possibilities to improve the sensitivity of quantum sensors and execute nontrivial quantum protocols in dense spin ensembles.

\section{Methods}

Samples preparation. The semi-insulating (SI) $4 \mathrm{H}-\mathrm{SiC}$ substrate with natural isotope content $\left(4.7 \%\right.$ of ${ }^{29} \mathrm{Si}$ and $1.1 \%$ of $\left.{ }^{13} \mathrm{C}\right)$ was purchased from Cree. The $6 \mathrm{H}$ and $4 \mathrm{H}-{ }^{28} \mathrm{SiC}$ samples, purified from the ${ }^{29} \mathrm{Si}$ isotope, were grown by the seeded physical vapour transport method ${ }^{34}$ with a ${ }^{28} \mathrm{Si}$ enriched precursor material on a $6 \mathrm{H}-\mathrm{SiC}$ substrate with natural isotope content. The ${ }^{29} \mathrm{Si}$ isotope abundance in these samples of $1 \%$ was established by the EPR spectroscopy (Supplementary Note and Supplementary Fig. 1). To create $\mathrm{V}_{\mathrm{Si}}$ centers, the $6 \mathrm{H}^{-28} \mathrm{SiC}$ and SI $4 \mathrm{H}-\mathrm{SiC}$ samples 
were irradiated with $2 \mathrm{MeV}$ electrons at temperatures close to room temperature to a total dose of $10^{18} \mathrm{~cm}^{-2}$. To create $\mathrm{V}_{\mathrm{Si}}$ centers in $4 \mathrm{H}_{-}{ }^{28} \mathrm{SiC}$, the sample was irradiated with 3-5 MeV neutrons at temperatures close to room temperature to a total dose of $10^{15} \mathrm{~cm}^{-2}$.

Two-frequency optically detected magnetic resonance (ODMR). A scheme of the experimental setup used in our ODMR/hole-burning experiments is presented in Supplementary Fig. 2. A 787.7 -nm diode laser (LD785-SE400 from Thorlabs) was pulsed using an acousto-optic modulator (AOM A\&A Opto-Electronic MT250-A02$800)$. The free space laser beam was coupled by several mirrors into a $50-\mu \mathrm{m}$ optical fiber, which transfers the light through a $\times 10$ microscope objective (Olympus LMPLN10XIR). The objective focused the beam on the sample surface with a spot diameter of approximately $10 \mu \mathrm{m}$. The laser power was about $10 \mathrm{~mW}$ at the sample surface. The PL was collected by the same $\times 10$ objective and filtered by an $850-\mathrm{nm}$ and $875-\mathrm{nm}$ long pass before it was coupled into a $600-\mathrm{nm}$ optical fiber. As a detector, a Si avalanche photodiode was used (APD120A from Thorlabs). The signal was processed by a lock-in amplifier (Signal Recovery DSP 7230). Radiofrequency was generated by a signal generator (Stanford Research Systems SG384) and amplified by an amplifier (Vectawave VBA1000-18) before being supplied to the sample by a $0.5-\mathrm{mm}$ copper-stripline the sample was placed on. In the hole burning experiments, the second radiofrequency was supplied by an identical Stanford Research Systems SG384 generator. The radio waves were modulated using RF-switches (Mini-Circuits ZASWA-2-50DR+) and a mixed before entering the amplifier using combiner (MiniCircuits ZFSC-2-4-S+). To apply the external magnetic field, a permanent magnet below the sample and Helmholtz coil was used.

Multipole decomposition. An ensemble of spin- $3 / 2$ centers is described by the $4 \times 4$ spin-density matrix $\rho$, which can be decomposed into a sum of the 16 basis matrices,

$$
\rho=\sum_{i=1}^{16} \operatorname{Tr}\left(\mathcal{M}_{i}^{\dagger} \rho\right) \mathcal{M}_{i} .
$$

The matrices $\mathcal{M}_{j}$ comprise three matrices of the spin dipole,

$$
\begin{aligned}
\mathcal{P}_{0} & =\left(\begin{array}{cccc}
\frac{3}{2 \sqrt{5}} & 0 & 0 & 0 \\
0 & \frac{1}{2 \sqrt{5}} & 0 & 0 \\
0 & 0 & -\frac{1}{2 \sqrt{5}} & 0 \\
0 & 0 & 0 & -\frac{3}{2 \sqrt{5}}
\end{array}\right), \\
\mathcal{P}_{+1} & =\left(\begin{array}{cccc}
0 & \sqrt{\frac{3}{10}} & 0 & 0 \\
0 & 0 & \sqrt{\frac{2}{5}} & 0 \\
0 & 0 & 0 & \sqrt{\frac{3}{10}} \\
0 & 0 & 0 & 0
\end{array}\right), \mathcal{P}_{-1}=\mathcal{P}_{+1}^{\dagger},
\end{aligned}
$$

five matrices of the spin quadrupole,

$$
\begin{aligned}
\mathcal{D}_{0} & =\left(\begin{array}{cccc}
\frac{1}{2} & 0 & 0 & 0 \\
0 & -\frac{1}{2} & 0 & 0 \\
0 & 0 & -\frac{1}{2} & 0 \\
0 & 0 & 0 & \frac{1}{2}
\end{array}\right), \mathcal{D}_{+1}=\left(\begin{array}{cccc}
0 & -\frac{1}{\sqrt{2}} & 0 & 0 \\
0 & 0 & 0 & 0 \\
0 & 0 & 0 & \frac{1}{\sqrt{2}} \\
0 & 0 & 0 & 0
\end{array}\right), \\
\mathcal{D}_{+2} & =\left(\begin{array}{llll}
0 & 0 & \frac{1}{\sqrt{2}} & 0 \\
0 & 0 & 0 & \frac{1}{\sqrt{2}} \\
0 & 0 & 0 & 0 \\
0 & 0 & 0 & 0
\end{array}\right), \mathcal{D}_{-1}=\mathcal{D}_{+1}^{\dagger}, \mathcal{D}_{-2}=\mathcal{D}_{+2}^{\dagger},
\end{aligned}
$$

seven matrices of the spin octupole,

$$
\begin{aligned}
\mathcal{F}_{0} & =\left(\begin{array}{cccc}
\frac{1}{2 \sqrt{5}} & 0 & 0 & 0 \\
0 & -\frac{3}{2 \sqrt{5}} & 0 & 0 \\
0 & 0 & \frac{3}{2 \sqrt{5}} & 0 \\
0 & 0 & 0 & -\frac{1}{2 \sqrt{5}}
\end{array}\right), \\
\mathcal{F}_{+1} & =\left(\begin{array}{cccc}
0 & \frac{1}{\sqrt{5}} & 0 & 0 \\
0 & 0 & -\sqrt{\frac{3}{5}} & 0 \\
0 & 0 & 0 & \frac{1}{\sqrt{5}} \\
0 & 0 & 0 & 0
\end{array}\right), \quad \mathcal{F}_{+2}=\left(\begin{array}{cccc}
0 & 0 & \frac{1}{\sqrt{2}} & 0 \\
0 & 0 & 0 & -\frac{1}{\sqrt{2}} \\
0 & 0 & 0 & 0 \\
0 & 0 & 0 & 0
\end{array}\right), \\
\mathcal{F}_{+3} & =\left(\begin{array}{cccc}
0 & 0 & 0 & -1 \\
0 & 0 & 0 & 0 \\
0 & 0 & 0 & 0 \\
0 & 0 & 0 & 0
\end{array}\right), \mathcal{F}_{-1}=\mathcal{F}_{+1}^{\dagger}, \mathcal{F}_{-2}=\mathcal{F}_{+2}^{\dagger}, \mathcal{F}_{-3}=\mathcal{F}_{+3}^{\dagger},
\end{aligned}
$$

and the diagonal matrix $\mathcal{I} / 2$, with $\mathcal{I}$ being the unit matrix. The above matrices form a complete orthogonal basis, $\operatorname{Tr}\left(\mathcal{M}_{i}^{\dagger} \mathcal{M}_{j}\right)=\delta_{i j}$.
Calculation of ODMR spectra. The density matrix describing an ensemble of spin centers satisfies the equation ${ }^{20}$,

$$
\frac{d \rho}{d t}=\frac{\mathrm{i}}{\hbar}[\rho, H]+G+(\dot{\rho})_{\mathrm{rel}},
$$

where $H$ is the Hamiltonian,

$$
H=D\left(S_{z}^{2}-\frac{5}{4}\right)+\hbar \gamma[\boldsymbol{B}+\tilde{\boldsymbol{B}}(t)] \cdot \boldsymbol{S},
$$

$\widetilde{\boldsymbol{B}}(t)=\sum_{\alpha=1,2}\left(\boldsymbol{B}_{\alpha} \mathrm{e}^{-\mathrm{i} \omega_{\alpha} t}+\right.$ c.c. $)$ is the magnetic field of the two-frequency (pump and probe) MW radiation, and $2 D$ is the zero-field splitting. Optical initialization of the spin centers is described by the term $G=\eta \mathcal{D}_{0} I_{0}$, where $\mathcal{D}_{0}$ is the spin quadrupole given by Eq. (6), $\eta$ is a material parameter $(\eta>0$ or $\eta<0$ depending on whether the $m_{S}= \pm 3 / 2$ or $m_{S}= \pm 1 / 2$ spin states are preferentially populated at optical excitation), and $I_{0}$ is the light intensity.

Spin relaxation is taken into account in Eq. (8) by the term

$$
(\dot{\rho})_{\text {rel }}=-\frac{\rho_{P}}{T_{p}}-\frac{\rho_{D}}{T_{d}}-\frac{\rho_{\mathcal{F}}}{T_{f}},
$$

where $\rho_{\mathcal{P}}=\sum_{k=-1}^{1} \operatorname{Tr}\left(\mathcal{P}_{k}^{\dagger} \rho\right) \mathcal{P}_{k}, \rho_{\mathcal{D}}=\sum_{k=-2}^{2} \operatorname{Tr}\left(\mathcal{D}_{k}^{\dagger} \rho\right) \mathcal{D}_{k}$, and $\rho_{\mathcal{F}}=\sum_{k=-3}^{3} \operatorname{Tr}\left(\mathcal{F}_{k}^{\dagger} \rho\right) \mathcal{F}_{k}$ are the dipole, quadrupole, and octupole contributions to the density matrix, respectively, $T_{p}, T_{d}$, and $T_{f}$ are the corresponding relaxation times.

The spin-density matrix $\rho$ found from Eq. (8) is used to calculate the spindependent correction to the PL intensity ${ }^{20}$

$$
\Delta \mathrm{PL} / \mathrm{PL}=\alpha \operatorname{Tr}\left(\mathcal{D}_{0} \rho\right),
$$

where $\alpha$ is a material constant. The result is then averaged over the ensemble of centers with slightly different values of the zero-filed splitting $D$, as described above.

Semi-classical rate equations. Equation (8) is simplified in the basis of the eigenstates of the Hamiltonian (9) without the MW field. Disregarding the off-diagonal components of the density matrix, we obtain the semi-classical rate equations for the populations $\rho_{i i}$ of the spin sublevels

$$
\frac{d \rho_{i i}}{d t}=\sum_{j} W_{i j}\left(\rho_{i i}-\rho_{j j}\right)+G_{i}-\sum_{j} R_{i j} \rho_{i j} .
$$

Here, $W_{i j}$ are the rates of spin transitions by the MW fields,

$$
W_{i j}=\sum_{\alpha} \frac{2 \delta \omega_{\alpha} \hbar^{2} \gamma^{2}\left|\boldsymbol{B}_{\alpha} \cdot S_{i j}\right|^{2}}{\left(\left|E_{i}-E_{j}\right|-\hbar \omega_{\alpha}\right)^{2}+\left(\hbar \delta \omega_{\alpha}\right)^{2}},
$$

$\delta \omega_{\alpha}$ are the halfwidths of the MW field spectra, which are supposed to have the Lorentzian shape, $G_{i}=\eta\left[\mathcal{D}_{0}\right]_{i i} I_{0}$, and $R_{i j}=R_{j i}$ are the component of the matrix of relaxation rates

$$
\begin{aligned}
R_{i j}= & \frac{1}{T_{p}} \sum_{k=-1}^{1}\left[\mathcal{P}_{k}\right]_{i i}\left[\mathcal{P}_{k}\right]_{j j}^{*}+\frac{1}{T_{d}} \sum_{k=-2}^{2}\left[\mathcal{D}_{k}\right]_{i i}\left[\mathcal{D}_{k}\right]_{j j}^{*} \\
& +\frac{1}{T_{f}} \sum_{k=-3}^{3}\left[\mathcal{F}_{k}\right]_{i i}\left[\mathcal{F}_{k}\right]_{j j}^{*} .
\end{aligned}
$$

For $\boldsymbol{B} \| z$, the components $R_{i j}$ have a particular simple form

$$
\begin{aligned}
& R_{+3 / 2,+3 / 2}=R_{-3 / 2,-3 / 2}=\frac{1}{20}\left(\frac{9}{T_{p}}+\frac{5}{T_{d}}+\frac{1}{T_{f}}\right), \\
& R_{+3 / 2,+1 / 2}=R_{-3 / 2,-1 / 2}=\frac{1}{20}\left(\frac{3}{T_{p}}-\frac{5}{T_{d}}-\frac{3}{T_{f}}\right), \\
& R_{+3 / 2,-1 / 2}=R_{-3 / 2,+1 / 2}=\frac{1}{20}\left(-\frac{3}{T_{p}}-\frac{5}{T_{d}}+\frac{3}{T_{f}}\right), \\
& R_{+3 / 2,-3 / 2}=\frac{1}{20}\left(-\frac{9}{T_{p}}+\frac{5}{T_{d}}-\frac{1}{T_{f}}\right), \\
& R_{+1 / 2,+1 / 2}=R_{-1 / 2,-1 / 2}=\frac{1}{20}\left(\frac{1}{T_{p}}+\frac{5}{T_{d}}+\frac{9}{T_{f}}\right), \\
& R_{+1 / 2,-1 / 2}=\frac{1}{20}\left(-\frac{1}{T_{p}}+\frac{5}{T_{d}}-\frac{9}{T_{f}}\right) .
\end{aligned}
$$

The $\mathrm{PL}$ correction is given by $\Delta \mathrm{PL} / \mathrm{PL}=\alpha \sum_{i}\left[\mathcal{D}_{0}\right]_{i i} \rho_{i i}$.

\section{Data availability}

The data supporting this study are available from the corresponding author upon reasonable request.

Received: 14 August 2018 Accepted: 6 March 2019 Published online: 11 April 2019

\section{References}

1. Awschalom, D. D., Bassett, L. C., Dzurak, A. S., Hu, E. L. \& Petta, J. R. Quantum spintronics: engineering and manipulating atom-like spins in semiconductors. Science 339, 1174-1179 (2013). 
2. Kaszlikowski, D. et al. Quantum cryptography based on qutrit Bell inequalities. Phys. Rev. A. 67, 656 (2003).

3. Lanyon, B. P. et al. Simplifying quantum logic using higher-dimensional Hilbert spaces. Nat. Phys. 5, 134-140 (2008).

4. Shlyakhov, A. R. et al. Quantum metrology with a transmon qutrit. Phys. Rev. A. 97, 022115 (2018).

5. Kues, M. et al. On-chip generation of high-dimensional entangled quantum states and their coherent control. Nature 546, 622-626 (2017).

6. Neeley, M. et al. Emulation of a quantum spin with a superconducting phase qudit. Science 325, 722-725 (2009).

7. Atatüre, M., Englung, D., Vamivakas, N., Lee, S.-Y. \& Wrachtrup, J. Material platforms for spin-based photonic quantum technologies. Nat. Rev. Mater. 3, 38-51 (2018)

8. Awschalom, D., Hanson, R., Wrachtrup, J. \& Zhou, B. B. Quantum technologies with optically interfaced solid-state spins. Nat. Photonics 12, 516-527 (2018)

9. Kraus, H. et al. Room-temperature quantum microwave emitters based on spin defects in silicon carbide. Nat. Phys. 10, 157-162 (2014).

10. Kimoto, T. \& Cooper, J. A. Fundamentals of Silicon Carbide Technology: Growth, Characterization, Devices and Applications. (John Wiley \& Sons, Singapore, 2014)

11. Falk, A. L. et al. Polytype control of spin qubits in silicon carbide. Nat. Commun. 4, 1819 (2013).

12. Biktagirov, T. et al. Polytypism driven zero-field splitting of silicon vacancies in 6H-sick. Phys. Rev. B 98, 195204 (2018).

13. Falk, A. L. et al. Electrically and mechanically tunable electron spins in silicon carbide color centers. Phys. Rev. Lett. 112, 187601 (2014).

14. Klimov, P. V., Falk, A. L., Christle, D. J., Dobrovitski, V. V. \& Awschalom, D. D. Quantum entanglement at ambient conditions in a macroscopic solidstate spin ensemble. Sci. Adv. 1, e1501015 (2015).

15. Widmann, M. et al. Coherent control of single spins in silicon carbide at room temperature. Nat. Mater. 14, 164-168 (2015).

16. Simin, D. et al. Locking of electron spin coherence above $20 \mathrm{~ms}$ in natural silicon carbide. Phys. Rev. B 95, 161201(R) (2017).

17. Nagy, R. et al. Quantum properties of dichrioic silicon vacancies in silicon carbide. Phys. Rev. Appl. 9, 034022 (2018).

18. Mizuochi, N. et al. Continuous-wave and pulsed EPR study of the negatively charged silicon vacancy with $\mathrm{S}=3 / 2$ and $\mathrm{C}_{3 v}$ symmetry in n-type $4 \mathrm{H}-\mathrm{SiC}$. Phys. Rev. B 66, 235202 (2002).

19. Carter, S. G., Soykal, Ö. O., Dev, P., Economou, S. E. \& Glaser, E. R. Spin coherence and echo modulation of the silicon vacancy in $4 \mathrm{H}-\mathrm{SiC}$ at room temperature. Phys. Rev. B 92, 161202 (2015).

20. Tarasenko, S. A. et al. Spin and optical properties of silicon vacancies in silicon carbide - a review. Phys. Stat. Sol. (b) 255, 1700258 (2018)

21. Kraus, $\mathrm{H}$. et al. Magnetic field and temperature sensing with atomic-scale spin defects in silicon carbide. Sci. Rep. 4, 5303 (2014).

22. Christle, D. J. et al. Isolated electron spins in silicon carbide with millisecond coherence times. Nat. Mater. 14, 160-163 (2015).

23. Soltamov, V. A., Soltamova, A. A., Baranov, P. G. \& Proskuryakov, I. I. Room temperature coherent spin alignment of silicon vacancies in $4 \mathrm{H}$ - and $6 \mathrm{H}-\mathrm{SiC}$. Phys. Rev. Lett. 108, 226402 (2012).

24. Sörman, E. et al. Silicon vacancy related defect in $4 \mathrm{H}$ and $6 \mathrm{H} \mathrm{SiC.} \mathrm{Phys.} \mathrm{Rev.} B$ 61, 2613-2620 (2000).

25. Riedel, D. et al. Resonant addressing and manipulation of silicon vacancy qubits in silicon carbide. Phys. Rev. Lett. 109, 226402 (2012).

26. Kehayias, P. et al. Microwave saturation spectroscopy of nitrogen-vacancy ensembles in diamond. Phys. Rev. B 89, 245202 (2014).

27. Mrózek, M. et al. Coherent population oscillations with nitrogen-vacancy color centers in diamond. Phys. Rev. B 94, 552 (2016).

28. Simin, D. et al. All-Optical dc nanotesla magnetometry using silicon vacancy fine structure in isotopically purified silicon carbide. Phys. Rev. X 6, 031014 (2016).
29. Simin, D. et al. High-precision angle-resolved magnetometry with uniaxial quantum centers in silicon carbide. Phys. Rev. Appl. 4, 014009 (2015).

30. Niethammer, M. et al. Vector magnetometry using silicon vacancies in $4 \mathrm{H}-\mathrm{SiC}$ under ambient conditions. Phys. Rev. Appl. 6, 034001 (2016).

31. Fang, K. et al. High-sensitivity magnetometry based on quantum beats in diamond nitrogen-vacancy centers. Phys. Rev. Lett. 110, 130802 (2013).

32. Fuchs, F. et al. Engineering near-infrared single-photon emitters with optically active spins in ultrapure silicon carbide. Nat. Commun. 6, 7578 (2015).

33. Muzha, A. et al. Room-temperature near-infrared silicon carbide nanocrystalline emitters based on optically aligned spin defects. Appl. Phys. Lett. 105, 243112 (2014).

34. Tairov, Y. M. \& Tsvetkov, V. F. Investigation of growth processes of ingots of silicon carbide single crystals. J. Cryst. Growth 43, 209-212 (1978).

\section{Acknowledgements}

This work was supported by the German Research Foundation (DFG) under Grants DY $18 / 13$ and AS $310 / 5$, by the ERA.Net RUS Plus program and the German Federal Ministry of Education and Research (BMBF) within project DIABASE. V.A.S. was supported by the Alexander von Humboldt-Foundation. A.V.P. and S.A.T. acknowledge the support by the RFBR \#17-02-00383 and the "BASIS" Foundation.

\section{Author contributions}

V.A.S., C.K., A.S., and A.N.A. performed the experimental work. A.V.P. and S.A.T. developed the theory. E.N.M. grew the samples. V.A.S., P.G.B., G.V.A., and V.D. conceived the experiments and supervised the project. All the authors contributed to analysis of the data, discussions and to the writing of the paper.

\section{Additional information}

Supplementary Information accompanies this paper at https://doi.org/10.1038/s41467 019-09429-x.

Competing interests: The authors declare no competing interests.

Reprints and permission information is available online at http://npg.nature.com/ reprintsandpermissions/

Journal peer review information: Nature Communications thanks the anonymous reviewers for their contribution to the peer review of this work. Peer reviewer reports are available.

Publisher's note: Springer Nature remains neutral with regard to jurisdictional claims in published maps and institutional affiliations.

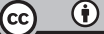

Open Access This article is licensed under a Creative Commons Attribution 4.0 International License, which permits use, sharing, adaptation, distribution and reproduction in any medium or format, as long as you give appropriate credit to the original author(s) and the source, provide a link to the Creative Commons license, and indicate if changes were made. The images or other third party material in this article are included in the article's Creative Commons license, unles indicated otherwise in a credit line to the material. If material is not included in the article's Creative Commons license and your intended use is not permitted by statutory regulation or exceeds the permitted use, you will need to obtain permission directly from the copyright holder. To view a copy of this license, visit http://creativecommons.org/ licenses/by/4.0/.

(C) The Author(s) 2019 